\title{
A New Issue
}

With this issue, Phenomenology + Pedagogy enters its second year. The growing pains of the initial "production" process have abated. You will notice some minor changes in the appearance of the journal. Its jacket size has grown a bit smaller to achieve easier portability as well as to cut paper waste. Some subtle design changes have also been made in order to maintain cordiality with our friends, the printers. Of course, we are striving to keep the essence of the journal intact. We look forward to your continued involvement, interest and support.

In this issue we are featuring a special theme section on Teaching and Doing Phenomenology. In upcoming issues we will pursue this interest further by sponsoring articles which aim to demonstrate, illustrate, and discuss the critical practice of such orientations. We invite your contributions. 